\title{
Device Not Accessible for Testing
}

National Cancer Institute

\section{Source}

National Cancer Institute. Device Not Accessible for Testing. NCI Thesaurus. Code C139463.

The actual device involved in the adverse event is not readily accessible for testing (e.g. remains implanted in patient). 